\title{
La decoración lítica de las Torres de Serranos de Valencia
}

The lithic decoration of the Serranos Towers of Valencia

\section{Concepción López González}

Universitat Politècnica de València, Valencia, Spain, mlopezg@ega.upv.es

\begin{abstract}
The apparent austerity of this powerful door of the Christian wall of the city of Valencia built in 1392 by Pere Balaguer, encloses a magnificent and rich decoration of carved stone. The intervention aimed at cleaning the stone promoted by the City Council, allowed an in-depth study of the ornamentation that had not been previously carried out. A graphic study of all the pieces of carved stone that decorate the Towers was made. The different types of decoration carved in stone were established according to three parameters: according to their function, according to the theme or ornamental motif and according to the type of size used. Likewise, comparative analyzes were established with other buildings constructed in the Crown of Aragon at the same time. This study showed the influences and collaborations between stonework workshops that worked in different geographical points.
\end{abstract}

Keywords: Torres de Serranos, gothic decoration.

\section{Introducción}

Las Torres de Serranos de Valencia comenzaron a construirse en el año 1392 al ser ampliada el área urbana de la ciudad. A raíz de este ensanchamiento se construyó una nueva muralla defensiva constituyendo las denominadas Torres de Serranos la puerta septentrional de la nueva cerca. En ese momento, finales del XIV y comienzos del XV, la ciudad vivía su siglo de oro. Su fértil agricultura, el desarrollo de una importante industria (cerámica, seda, terciopelo, pieles, etc.) así como un floreciente comercio a través de su puerto marítimo con el resto de ciudades del Mediterráneo convierten a Valencia en una próspera ciudad que desea demostrar a sus visitantes su opulencia a través de la grandiosidad de sus edificios públicos. "Los maestros compiten entre ellos a la hora de buscar nuevas soluciones constructivas; se exploran originales procedimientos geométricos conducentes al tra- zado de arcos, bóvedas y escaleras" (López, 2012, p. 166).

Este afán de embellecimiento de la ciudad por parte de los Jurados quedó reflejado en la construcción de la Puerta septentrional por la que entraban los visitantes provenientes del resto de territorios peninsulares de la Corona de Aragón tras cruzar el río Turia.

Su construcción fue encargada al maestro cantero Pere Balaguer molt sabut en l'art de la pedra. Este mismo maestro participó posteriormente en la construcción de la iglesia de Santa Catalina (1406-1422), en el cimborrio de la Catedral (1410) y en su campanario denominado Miguelete (1414).

Pere Balaguer dirigió la totalidad de la obra, incluida la ornamentación en piedra, como demuestran las múltiples visitas a diferentes cante- 
ras para elegir el material adecuado a cada uso (Serra, 2003, p. 15). No hay que olvidar que los maestros de este periodo se formaban en diferentes disciplinas relacionadas con el proceso constructivo y eran habituales las colaboraciones y vinculaciones entre maestros. El propio Pere Balaguer trabajó con Bertomeu Coscollá, orfebre, en el segundo cuerpo del cimborrio de la catedral y con Martí Lobet como escultor en el Miguelete.

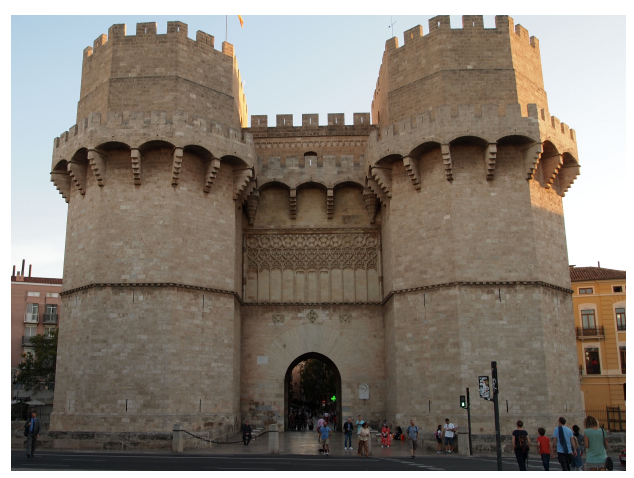

Fig. 1. Fachada septentrional de las Torres de Serranos en la actualidad (Concepción López).

El estudio de la criptografía (existen más de 3000 marcas de cantería) ha demostrado que intervinieron 26 talleres en su construcción aunque 7 fueron los que más trabajaron en ella (Cervera, Mileto, 2003, p. 128). Pere Nicolau pintó y doró en 1394 las letras de la Puerta y las claves de las salas superiores.

Las obras finalizaron en marzo de 1398, seis años después de su comienzo (Carboneres, 1873, p. 9).

\section{Objetivo y metodología de la investigación}

Anteriormente al trabajo de investigación realizado no existía una documentación rigurosa relativa a las piezas esculpidas que decoran las Torres de Serranos. Gracias a la iniciativa del Ayuntamiento de la ciudad (2000) destinada a la realización de un estudio previo anterior a la limpieza de la piedra fue posible realizar el análisis de la decoración de este importante monumento. El arqutiecto coordinador de la intervención fue francisco Cervera, mientas que la coordinación de la toma de datos tradicional para el levantmaiento de planos y la catalogación de las piezas decoradas fue llevada a cabo por Concepción López González. El objetivo principal del estudio fue la catalogación de todas y cada una de la piezas esculpidas en piedra que decoran las Torres de Serranos.

Para ello, y aprovechando el montaje de andamios que permitía el acceso directo a todas las piezas escultóricas, se realizó un levantamiento de planos conjuntamente con un reportaje fotográfico de cada una de ellas, situando cada pieza sobre un plano general.

La toma de datos del levantamiento de planos tuvo que acometerse de forma diferente al habitual. Al tratase de formas escultóricas fue necesario emplear una variable gráfica que en los croquis tradicionales no suele tenerse en cuenta: el uso de las sombras.

Se utilizó la línea, sensibilizada a través de valores de grosor y peso que jerarquizan la intencionalidad de la representación por poseer una gran variedad de niveles de refinamiento y significado, para definir contorno y aristas en las secciones. Sin embargo, los alzados y plantas fueron tratados mediante el uso del claroscuro, es decir, de la luz y la sombra dando un carácter realista o evocador a la representación. La percepción de la forma arquitectónica cambia según la posición de la luz. La luz resalta la volumetría debido a las sombras que se producen, poniendo de manifiesto los juegos volumétricos (López, 2009, p. 55). De esta forma los elementos escultóricos pudieron ser representados siguiendo un criterio de semejanza.

Para realizar un análisis sistemático se establecieron dos ámbitos de estudio bien diferenciados: La ornamentación que decora el exterior de las Torres y la que adorna el interior. Esta diferenciación se debe a dos motivos: por un lado se establece un marco espacial y por otro se evidencia una diferenciación formal entre uno y otro.

A su vez, cada una de las piezas decoradas de estos dos ámbitos fue clasificada según su ubicación. En el exterior se establecieron tres localizaciones: Tracería ciega sobre la portada de 
medio punto en el cuerpo central en su fachada recayente al río para ser admirada por todo el que llega a la ciudad; cornisa que recorre el perímetro de las Torres a un tercio de su altura y las ménsulas de apoyo de la barbacana. En el interior las piezas fueron localizadas atendiendo a su ubicación en la torre oriental, la torre occidental y el cuerpo central, y a su vez, según se encontraran en planta baja, primera o segunda. De este modo, todas y cada una de las piezas existentes en la Torres fueron localizadas y analizadas a través del dibujo.
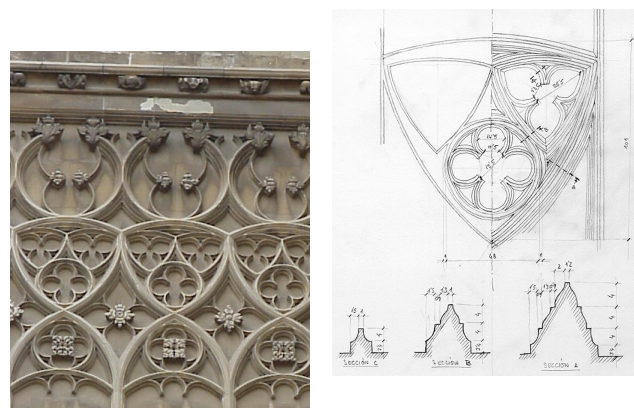

Fig. 2. Detalle de la tracería sobre la puerta. Fotografía y croquis (Concepción López).

Posteriormente, y tras el análisis formal correspondiente, se establecieron clasificaciones atendiendo al motivo de la decoración y al tipo de talla.

De todo ello fue posible extraer conclusiones relativas a la diversidad de la autoría, a los motivos ornamentales utilizados y a las influencias exteriores reflejadas en las piezas esculpidas.

\section{Resultados: catalogación de la decoración}

La potente imagen defensiva de las Torres de Serranos, de grandes dimensiones, perfecta factura, armoniosas proporciones y elegantes formas, no hace presagiar la hermosa y variada decoración que las ornamenta. El proyecto de investigación que llevamos a cabo puso en evidencia el intenso trabajo escultórico realizado en esta magnífica puerta.

\subsection{La decoración exterior}

Las piezas líticas esculpidas que ornamentan las fachadas de las Torres de Serranos se encuen- tran, como vimos, distribuidas en tres ámbitos: la lacería, la cornisa y las ménsulas de la barbacana.

A) La lacería fue sustituida en su mayor parte a finales del siglo XIX aunque se puede afirmar, tras la observación de documentos fotográficos realizados antes y después de la intervención, que las piezas sustituidas reproducen fielmente el modelo gótico original. Esta reforma fue acometida por José Aixa, reconocido como restaurador artístico en 1892. De esta importante intervención no se tienen documentos gráficos y muy pocos documentos escritos por lo que hasta la actualidad se sabía poco de ella.

De la antigua decoración sólo se conservan dos piezas esculpidas en los ángulos superiores y parte de los pináculos que flanquean la tracería. Estas piezas están recubiertas por un estrato de color rojo que, con toda seguridad, recubrió originalmente la totalidad de la tracería. La tracería está compuesta por cuatro alturas de decoración siendo el tamaño de las piezas de $21 \times 21 \mathrm{~cm}$ excepto en el tercer nivel que son de $15 \times 15 \mathrm{~cm}$. Se han catalogado 15 tipologías diferentes de piezas labradas con motivos vegetales, que se repiten de forma aleatoria, sin orden compositivo.

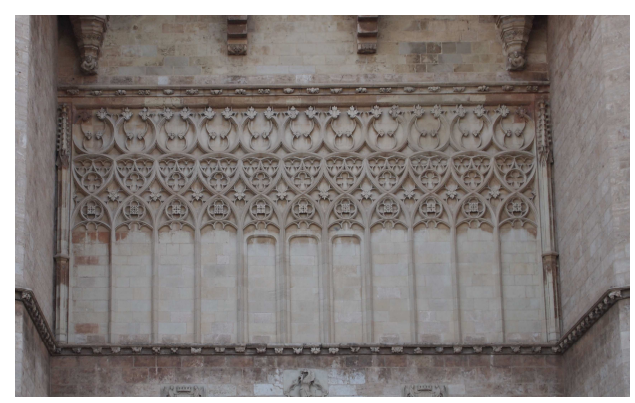

Fig. 3. Tracería Ciega. (Concepción López).

Se distribuyen del siguiente modo:

En la fila inferior las piezas están labradas con motivos vegetales a base de hojas de borde hendido en unos casos y de borde entero en otros. Existen en total tres tipologías diferentes. En la siguiente fila los motivos son vegetales de hojas palmeadas de borde hendido similar al sasafrás que nacen de un elemento circular. Se distinguen 
tres tipologías diferentes. En la fila superior las piezas están colocadas giradas $45^{\circ}$ como remate final de las filigranas de la lacería de este nivel. (Fig. 3).

Encontramos cinco tipologías diferentes basadas en hojas palmeadas de borde hendido que surgen de un ovoide irregular central.
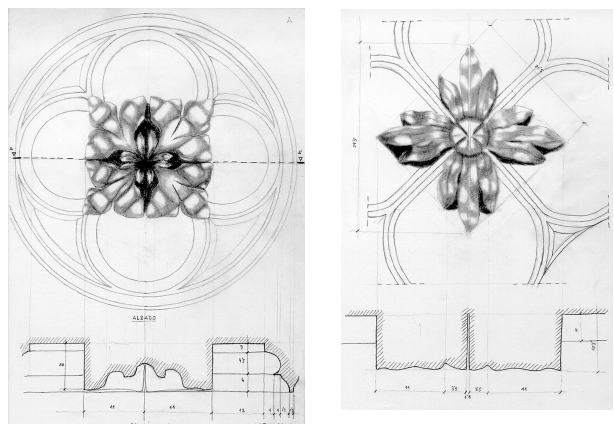

Fig. 3. Croquis de piezas labradas de la tracería del primer y segundo nivel (Concepción López).

Las piezas de la fila más alta son similares a las del nivel anterior pero colocadas en vertical y de mayor tamaño. Sobre la tracería discurre una cornisa con piezas labradas de motivos zoomorfos a base de rostros de animales (probablemente murciélagos) de los que salen hojas de la boca. Existen cuatro tipologías diferentes.

B) La cornisa recorre todo el perímetro de las Torres a un tercio de su altura. Está formada por una imposta con molduración gótica sobre la que se destacan 190 piezas decoradas de $23 \times 14 \mathrm{~cm}$ aproximadamente. Todas ellas se describen en unas fichas creadas para el inventario y localizadas mediante un esquema general de cada uno de los alzados dónde aparece la pieza (Fig. 4).

La torre oriental contiene 83 piezas labradas con 28 tipologías diferentes. La torre occidental dispone de 82 piezas de 15 tipologías diferentes, de las cuales 8 no se repiten a lo largo del resto de la cornisa. En el cuerpo central existen 25 piezas labradas, entre las que se advierten 11 tipologías diferentes de las cuales 2 no se repiten en el resto de la cornisa.

C) La barbacana que recorre el perímetro de la fachada septentrional apoya sobre 26 ménsulas escalonadas cuya base está formada por una pie- za esculpida con motivos vegetales, a excepción de las 4 ménsulas situadas en el inicio y final de la barbacana en cada torre, de mayores dimensiones y forma troncopiramidal invertida de planta octogonal y profusamente decorada a base de molduraciones labradas en la piedra.

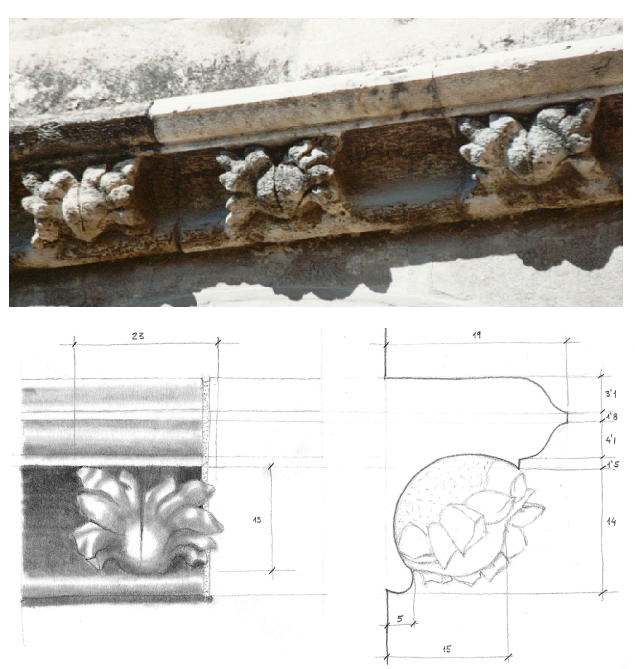

Fig. 4. Fotografía y croquis de pieza labrada de la cornisa (Concepción López).

Se distribuyen de la siguiente manera:

En la torre occidental existen 10 canes pequeños y uno grande en el extremo. Casi todos fueron sustituidos en la reforma del XIX a excepción de los tres del alzado norte. Los de esquina siguen la misma tipología a base de dos hojas de borde hendido que se unen en el centro. Los otros siete canes reemplazados siguen seis tipologías diferentes basadas en dos hojas de borde hendido en los extremos y cubiertas en la parte central por otra hoja palmeada. En algunos casos las hojas extremas son sustituidas por granadas abiertas.

En la torre oriental existen 10 tipologías diferentes, es decir, no se repite ningún can decorado. Sin embargo, tres de los canes de ellos siguen tipologías que aparecían en la torre occidental. En esta torre no se conserva ningún can original.

En el cuerpo central existen 2 ménsulas escalonadas y otras dos octogonales piramidales. Las dos primeras tienen tipologías diferentes y no se repiten en las torres. Las piramidales están deco- 
radas en sus esquinas superiores por dos hombres con túnica. La más próxima al muro es original y la otra fue sustituida a principios de siglo. La parte inferior del tronco de pirámide está decorada con otro hombre con túnica también de comienzos de siglo (Fig. 5).
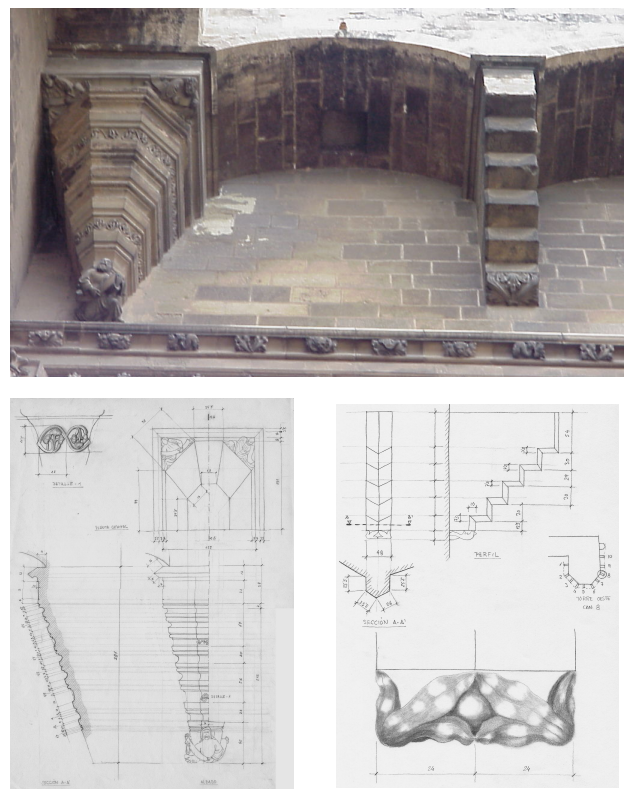

Fig. 5. Fotografía y Croquis de ménsula troncopiramidal y escalonada de la barbacana (Concepción López).

\subsection{La decoración interior}

Las piezas de piedra labrada en el interior de las Torres de Serranos se encuentran en los canes de arranque de los nervios de las bóvedas de crucería. Existen 58 arranques decorados en todo el conjunto arquitectónico de los cuales 16 , situados en las estancias de planta baja de las dos torres, han sido sustituidos recientemente con piezas prefabricadas con moldes y mortero, por lo que no han sido incluidos en esta catalogación.

De los 42 canes restantes, 2 están destrozados debido a la intervención destinada a convertir las Torres en cárceles cerrando los arcos abiertos que recaen sobre la plaza de los Fueros por lo que tampoco han sido considerados.

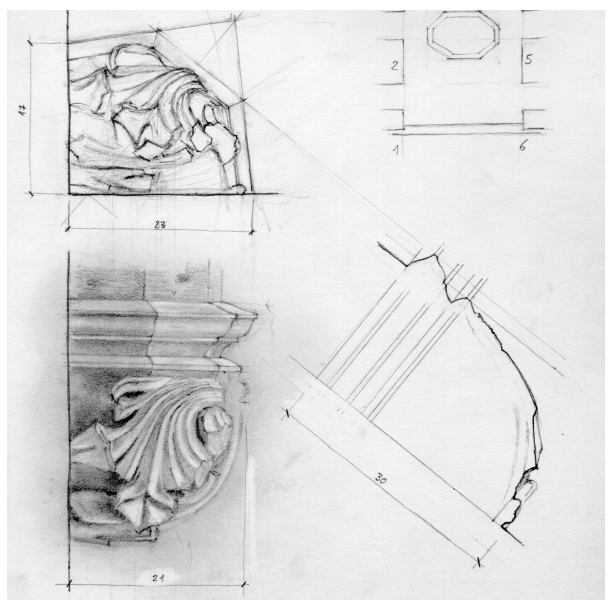

Fig. 6. Can de arranque de nervios del primer piso del cuerpo central. Tipología geométrica (Jesús GarcíaHerrero).

Tras el estudio de los 40 restantes se han podido establecer siete tipologías diferentes:

1) Entrelazada: basada en hojas palmeadas de borde hendido donde las hojas de los extremos, similares a las de acónito, se retuercen y entrelazan en el centro del can bajo una hoja central palmeada de borde hendido que recuerda la hoja de arce. Esta tipología es la más complicada en sus formas debido a la enmarañada disposición de las hojas (Fig. 7).

2) Geométrica: donde las hojas abandonan la suavidad de formas utilizando una talla más geométrica, buscando curvaturas más simples y aristas más vivas (Fig. 6).

3) Suave: las hojas palmeadas de borde entero adquieren formas suaves y ondulantes, de superficies más extensas y lisas (Fig. 8).

4) Nervada: basada en hojas parecidas a las de la tipología anterior pero con los nervios notablemente más marcados (Fig. 9).

5) Labra de un rostro humano de cuya boca salen dos hojas, como el denominado "green man" inglés. Sólo existe un can de este tipo en la planta segunda de la torre occidental (Fig. 10).

6) Labra de animales mitológicos como dragones. Sólo se encuentran en canes de esquina (Fig. 11). 
7) Labra animales alados similares a las águilas. Sólo aparece uno en la segunda planta de la torre oriental.
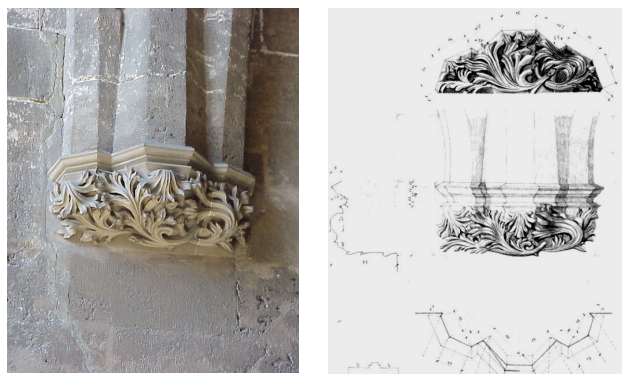

Fig. 7. Fotografía y dibujo de can en planta primera del cuerpo central. Tipo entrelazado (Concepción López).
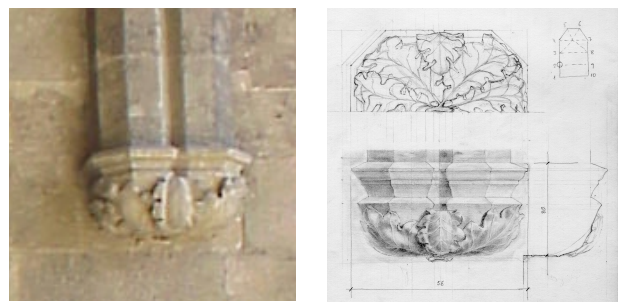

Fig. 8. Fotografía y dibujo de can en planta segunda de la torre occidental. Tipología suave. (Concepción López).
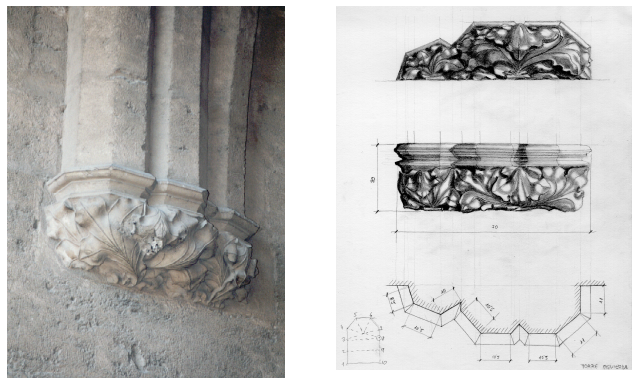

Fig. 9. Fotografía y dibujo de can en planta segunda de la torre occidental. Tipo nervado (Concepción López).
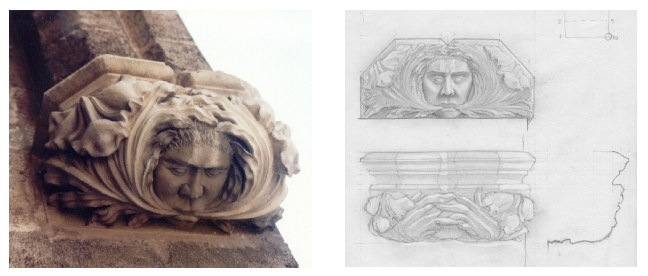

Fig. 10. Fotografía y dibujo de can con rostro humano, green man, en planta segunda de la torre occidental. (Concepción López).
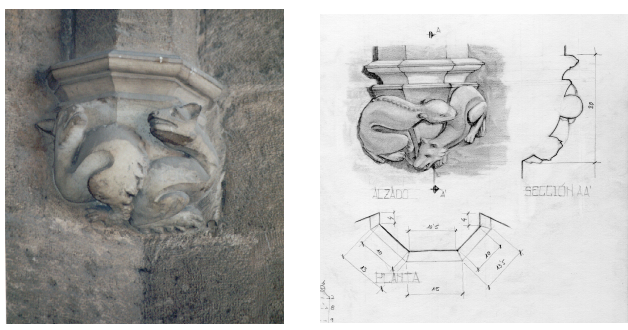

Fig. 11. Fotografía y dibujo de can con dragones en planta segunda de torre oriental. (Concepción López).

\section{Conclusiones}

Podemos concluir que la profusa y equilibrada decoración exterior de las Torres de Serranos supone un derroche de imaginación donde muy pocas piezas labradas en la piedra se repiten, donde aparece una gran variedad de tipologías diferentes y donde la métrica de las piezas modernas difiere notablemente de las que se corresponden con piezas originales.

Respecto a la labra de los sillares del interior de las Torres, se puede apreciar la mano de diversos artífices como evidencia la notable diferenciación, no sólo de los motivos ornamentales, sino también de la forma de tallar la piedra.

Los motivos elegidos, tanto en la decoración interior como en la decoración exterior, ya fueron utilizados por Reinard des Fonoll, cantero inglés que introduce el gótico florido en la península, en el claustro del monasterio de Santes Creus (1332-1341).

El perro esculpido en una de las pilastras de la escalera de acceso al primer piso de las Torres de Serranos también lo encontramos en los sepulcros de las reinas como símbolo de fidelidad, de guardián y de guía.

Los animales alados de los capiteles del claustro de Santes Creus se repiten en los canes de arranques de los nervios de la segunda planta como símbolos del bien según la simbología medieval.

Todo ello hace suponer que Reinard de Fonoll tuvo una clara influencia sobre Pere Balaguer. Probablemente a raíz del viaje que los Jurados de la ciudad le pagaron para que viera otras torres y puertas. De este modo, el gótico florido inglés hace su aparición en Valencia. 


\section{Bibliography}

Carboneres i Quiles, M. (1873). Nomenclátor de ls puertas, calles y plazas de Valencia, Imprenta del Avisador Valenciano, París-Valencia reimp. 1980, Valencia.

Cervera Arias, F; Mileto, C. (2003). "El estudio de las Torres de Serranos", in Las Torres de Serranos. Historia y Restauración, Ayuntamiento de Valencia, Valencia.

López González, C. (2009). La interpretación gráfica de la obra arquitectónica, Arquitectura y Humanidades. Architectum. México, pp. 36-57.

López González, C. (2012). "El buen oficio frente al concurso. Los maestros canteros valencianos del siglo XV”, XIV Congreso EGA, Universidad de Valladolid, Oporto, pp. 165-170.

Serra Desfilis, A. (2003). "El portal de los serranos en los siglos XIV y XV", in Las Torres de Serranos. Historia y Restauración, Ayuntamiento de Valencia, Valencia. 
